Piloterfahrungen mit einem Nachhaltigkeits-Audit

\title{
Integration von sich verändernden Umwelten
}

\author{
In den letzten Jahren wurden zahlreiche Ansätze entwickelt, die die Idee des \\ nachhaltigen Wirtschaftens in Unternehmen integrieren sollen. Ziel ist es, \\ neben der ökonomischen Seite auch ökologische und soziale Aspekte unterneh- \\ merischen Handelns aufzuzeigen. Dies wird notwendig, da sich das Umfeld der \\ Unternehmen verändert hat. Das neve Konzept des Nachhaltigkeits-Audits bie- \\ tet hierauf eine vielversprechende Antwort, wie erste Umsetzungserfahrungen \\ bei einem ökologischen Finanzdienstleister zeigen.
}

$\mathrm{D}$ Von Alexandra Lux, Claudia Empacher und Thomas Kluge ie Komplexität der Anforderungen und Zwänge, denen sich ein Unternehmen gegenüber sieht, ist sprunghaft gewachsen. Dies macht es notwendig, die eindimensionale Betrachtungsweise, wie sie beispielsweise das Umwelt- oder Qualitätsmanagement vorsieht, zu erweitern. Gefragt sind Ansätze, die Komplexität nicht reduzieren, aber dennoch einen produktiven Umgang mit ihr ermöglichen. Die bisherigen Ansätze zielen im Wesentlichen auf eine erweiterte Unternehmenskommunikation $a b$, doch eine Überführung dieser in ein Managementsystem findet nicht in ausreichendem Maße statt.

Vor diesem Hintergrund wurde das Nachhaltigkeits-Audit entwickelt, welches auf eine Verzahnung zwischen den Bereichen Betriebsökologie, Produktangebot, betriebsinterne Situation und Außenwirkung zielt. Konzeptionell werden die bereits bestehenden und zur Reife gebrachten prozessorientierten betriebsökologischen Formen des Öko-Audits mit einem Produkt- sowie SozialAudit zu einem Nachhaltigkeits-Audit zusammengefasst. Kern des Ansatzes ist die Analyse der Stärken und Schwächen eines Unternehmens in jedem dieser drei Audit-Teile sowie der Interdependenzen zwischen Problemfeldern und Lösungspotenzialen, um insgesamt einen kontinuierlichen Verbesserungsprozess zu initiieren. Durch die Offenlegung der Zusammenhänge zwischen den verschiedenen Ebenen eines Problems sollen verschiedene Steuerungsmöglichkeiten entdeckt und eine Balance gefunden werden. Das Ergebnis dieses aktiven Prozesses kann nicht von vornherein bestimmt werden. Dennoch sollte innerhalb des Unternehmens Einigkeit über die möglichen Ent- wicklungslinien zur Problemlösung bestehen, innerhalb derer Kompromisse gefunden, Prioritäten gesetzt und neue Stärken herausgebildet werden können (1).

Erste praktische Erfahrungen mit dem Konzept Nachhaltigkeits-Audit konnten mit einem ökologisch orientierten Finanzdienstleistungsunternehmen gemacht werden. Dieses Unternehmen befindet sich durch den Zuwachs an KundInnen und Produktangeboten wie auch an MitarbeiterInnen in einer spezifischen Phase der Neuordnung. Diese betrifft Strukturen und Managementprozesse, aber auch eine neue (ökologische) Profilierung und Positionierung nach außen. Es ergibt sich ein Problemgefüge, das einerseits betriebsspezifisch ist, andererseits aber auch Ausdruck der modernen, avancierten Organisationsstrukturen. Letztere zeichnen sich durch ein hohes Maß an Selbstorganisation, Verantwortungsdelegation an die MitarbeiterInnen und flachen Hierarchien aus. Dies macht die Organisation zu einem empfindlichen Gebilde, das auf Impulse von außen sehr sensibel reagieren kann. Hierin bieten sich viele Chancen zur Anpassung an ein sich veränderndes Umfeld, aber es können auch neue Probleme und Konflikte entstehen.

\section{Vom Konzept zur Umsetzung: Prozesse ...}

In Anlehnung an das Vorgehen bei der Durchführung eines Öko-Audits nach der EG-Öko-AuditVerordnung (EMAS) wird die Betriebsökologie untersucht. Die direkten Umweltwirkungen (vor allem Energie-, Wasser-, Papierverbrauch, Abfallaufkommen und Kohlendioxid-Emissionen) werden hier erfasst, beschrieben und bewertet. Im konkreten Fall wurde der Leitfaden für die Erstel- lung von Umweltbilanzen in Banken des Vereins für Umweltmanagement (2) berücksichtigt, da es so möglich wurde, die ermittelten Kennzahlen mit jenen zu vergleichen, die andere Banken in ihren Umweltberichten veröffentlichten. Dieser Branchen-Benchmark zeigte, dass die durch den Betrieb induzierten Umweltauswirkungen vergleichsweise gering und weitgehend optimiert sind, auch wenn sich in einigen Feldern, zum Beispiel beim Papierverbrauch, noch Handlungsbedarf erkennen ließ.

\section{D... Produkte ...}

Das Produkt-Audit nimmt die Idee der novellierten EMAS-Verordnung (3) auf, Produkte mit in die Bewertung der ökologischen Wirkungen eines Unternehmens zu integrieren. Durch die Prüfung der Glaubwürdigkeit und die Erweiterung um soziale Aspekte geht es aber einen Schritt weiter. Um das Produktangebot und damit auch die Außenwirkung des Pilotunternehmens bewerten und analysieren zu können, wurden hier die (indirekten) ökologischen Wirkungen und sozialen Aspekte der gesamten Produktpalette einbezogen. Sie wurden an den unternehmenseigenen Ansprüchen an nachhaltig(er)es Wirtschaften, zum Beispiel in Form des Unternehmensleitbildes, und den geltenden rechtlichen Regelungen geprüft. Darüber hinaus wurde auch hinterfragt, ob das Leitbild im Alltagsgeschäft eingehalten wurde und ob dies nachvollziehbar dokumentiert ist.

Grundlage für die Bewertung bildeten im Pilotunternehmen sowohl interne als auch veröffentlichte Schriften, aber auch Gespräche mit Verantwortlichen. Es konnte festgestellt werden, dass ein gut ausgebauter Bestand von ökologisch und sozial orientierten Produkten vorliegt, beispielsweise ökologisch orientierte Fonds oder die Verbindung von Baufinanzierung mit ökologischen und sozialen Mindestanforderungen. Es konnten aber auch erste Abschätzungen getroffen werden, welche Produktlinien weiter auszubauen bzw. neu aufzubauen sind. Hier spielte auch das Zusammenwirken mit den Einschätzungen der MitarbeiterInnen, die im Kundenkontakt stehen, eine Rolle.

\section{>... Soziales ...}

Das Sozial-Audit kann als sensibles Sensorium gesehen werden, das die Befindlichkeiten der Anspruchsgruppen des Unternehmens (Stakeholder) aufnimmt und umfangreicher als bisherige Partizipationsmethoden integriert (4). Bei der Umsetzung im Pilotunternehmen wurde aus Grün- 
den der Praktikabilität zunächst nur auf die Gruppe der MitarbeiterInnen eingegangen. Es wurde Einigung darüber erzielt, dass weitere Gruppen in den folgenden Audit-Zyklen mit einbezogen werden. Es wurden mit jeder MitarbeiterIn qualitative Einzelinterviews durchgeführt, da das Unternehmen mit ca. 80 MitarbeiterInnen eher klein ist. Die Fragen zielten im Wesentlichen auf

die Einstellung zu positiven bzw. negativen Aspekten ihrer Arbeit,

- Arbeitszeit und Arbeitsbelastung,

Verhältnis Arbeit - Freizeit,

Entscheidungen und Kommunikation,

- Führung und Organisationsstrukturen,

geschlechtsspezifische Aspekte,

- Außenwirkung sowie

- Unternehmenskultur/ -leitbild.

In den Fragestellungen wurde der Bezug zu ökonomischen und ökologischen Aspekten hergestellt, um hier die Verbindung zu den anderen Audit-Teilen zu gewährleisten.

Im Pilotunternehmen konnte festgestellt werden, dass sich die meisten MitarbeiterInnen sehr stark mit dem Unternehmensleitbild und der damit verbundenen Geschäftspolitik identifizieren können und diese unterstuitzen. Um zu einem Unternehmen zu kommen, in dem Selbstorganisation und Übernahme von Verantwortung durch die MitarbeiterInnen voll zum tragen kommen, hatte man sich bei Gründung für eine Organisation mit flachen Hierarchien entschieden. Diese Entscheidung hat Einfluss auf die Bedingungen für Führung und Leitung und schafft eigene Entwicklungsdynamiken.

\section{>... und Gender}

Parallel zu dieser Ablauforganisation wird stark auf ein gleichberechtigtes Zusammenarbeiten von Männern und Frauen geachtet. Für die MitarbeiterInnen-Ebene wurde dieses Ziel als weitestgehend umgesetzt bewertet. Aber auf der Managementebene waren wie in vielen Unternehmen weit weniger Frauen als Männer zu finden. Die Befragung gab Aufschluss darüber, warum auf Führungsebene das Ziel der Gleichberechtigung nicht umzusetzen ist: Das dort gelebte Führungsbild wird von den MitarbeiterInnen als maskulin geprägt wahrgenommen; es ist für Frauen weniger erstrebenswert, Leitungspositionen zu übernehmen - ungeachtet von Quotierungen und Qualifikationsmaßnahmen. Es wurde deutlich, dass eine neue Aufgabenübertragung und Verantwortungsverteilung zwischen Männern und Frauen erreicht werden muss - und das sowohl im betrieblichen als auch im privaten Bereich. Das Unternehmen sollte Strukturen schaffen, die Anreize für beide Geschlechter zur Übernahme von Führungsverantwortung bieten und somit ein neues Führungsbild ermöglichen. Dies könnte auch Wirkung auf die Motivation der MitarbeiterInnen haben. Es böten sich Möglichkeiten für Effizienzsteigerungen in den Geschäftsabläufen, die auch Einfluss auf die Profilierung nach außen, die Produktgestaltung, das Firmenimage und die Bindung neuer KundInnengruppen haben.

\section{Umgang mit Zielkonflikten}

Bereits ein erstes Screening der Ergebnisse des Produkt- und Sozial-Audits sowie der Prüfung der Betriebsökologie zeigte deutlich, wie diese Ebenen zusammenhängen. Folgendes Beispiel verdeutlicht dies: Es wurde ein hoher Papierverbrauch festgestellt. Aus Sicht der Betriebsökologie ist dies zunächst negativ zu bewerten, andererseits wurde von den MitarbeiterInnen gefordert, dass sie besser über Vorgänge und Entscheidungen innerhalb des Unternehmens informiert werden wollen. Es ließ sich somit ein Konflikt zwischen der ökologischen und sozialen Dimension erkennen. Die Entscheidung für die Verbreitung von Informationen über elektronische Medien wie das Intranet wiederum könnte zu einer gesteigerten Belastung der MitarbeiterInnen durch vermehrte Bildschirmarbeit führen, was den Konflikt nur noch verstärkt. Es müssen somit geeignete Lösungen zur Bündelung der Informationsflüsse gefunden werden, die transparent machen, welcher Personenkreis welche Informationen benötigt und auf welchem Wege erhält. Das hierzu notwendige Wissensmanagement hätte dann auch Schnittstellen zum Qualitätsmanagement, zum Dokumentationswesen, etc.

Diese Verzahnungen zeigen, wie ein Problem von der Betriebsökologie (Absenkung des Papierverbrauchs) über Bereiche der Arbeitsplatzgestaltung (Transparenz, Bildschirmarbeit) bis hin zur Produktökologie (Qualitätssicherung, Dokumentation) und zur Organisation des Qualitätsmanagements reicht und wie leicht Reibungspunkte entstehen können, die betriebliche Abläufe und Humanressourcen beeinflussen.

\section{- Erfahrungen sind übertragbar}

Das beschriebene Vorgehen stellt einen möglichen Weg der Umsetzung dar, der auf ein Unternehmen abgestimmt ist. Doch die im Konzept Nachhaltigkeits-Audit enthaltenen Prozessstandards können auf spezifische, unternehmerische
Bedürfnisse angepasst werden. Das Kernset des Nachhaltigkeits-Audits besteht aus einer Bestimmung der subjektiven Ansprüche der Stakeholder, der Prüfung der Produkte hinsichtlich ihrer sozialen und ökologischen Aspekte mittels eines Kriterienkatalogs, der auf das Produktangebot zugeschnitten ist, und der betriebsökologischen Untersuchung. Aus der Analyse der Ergebnisse der einzelnen Audit-Teile kann ein Stärken-Schwächen-Profil des Unternehmens abgeleitet werden, das dann in ein prozessorientiertes, auf nachhaltiges Wirtschaften ausgerichtetes Managementsystem überführt wird. Die Lösung der so erkennbaren Probleme wird durch eine zyklische Beobachtung und prozesshafte Vernetzung von Veränderungen erreicht. Diese Prozessstandards lassen genügend Freiraum für individuelle, unternehmensspezifische Anpassungen, die der Unterschiedlichkeit von Unternehmenstypen hinsichtlich Branchen, Größe, Produktpalette etc. gerecht werden.

\section{Anmerkungen}

(1) In Analogie zum sogenannten Leitplankenkonzept in der Nachhaltigkeitsdiskussion; vgl. Becker, Egon et al.: Sustainability: A Cross-Disciplinary Concept for Social Tranformations, MOST Policy Papers 6, UNESCO. Paris 1997. (2) Verein für Umweltmanagement in Banken, Sparkassen und Versicherungen (Hrsg): Bilanzierungsgrundsätze und -richtlinien für betriebliche Umweltkennzahlen der Finanzdienstleister mit Standardkontenrahmen. Bonn 1997.

(3) Europäischer Rat: Gemeinsamer Standpunkt des Rates im Hinblick auf den Erlass der Verordnung des Europäischen Parlaments und des Rates über die freiwillige Beteiligung von Organisationen an einem Gemeinschaftssystem für das Umweltmanagement und die Umweltbetriebsprüfung (EMAS) - Entwurf vom 11. Februar 2000. Brüssel. Vgl. hierzu auch den Beitrag von Pape auf S. 6-7. (4) Vgl. Empacher, Claudia/ Kluge, Thomas: Die Integration der sozialen Dimension in eine nachhaltige Unternehmenspolitik. In: UmweltWirtschaftsForum, 7. Jg., H. 1/1999, S. 87-91.

\section{Die Autorlnnen}

Alexandra Lux, Claudia Empacher und Thomas Kluge sind wissenschaftliche MitarbeiterInnen am Institut für sozial-ökologische Forschung (ISOE).

Kontakt: ISOE, Hamburger Allee 45, 60486 Frankfurt/Main. Tel. 069/ 7076919-27, Fax -11, E-mail: lux@isoe.de 
(c) 20I0 Authors; licensee IÖW and oekom verlag. This is an article distributed under the terms of the Creative Commons Attribution Non-Commercial No Derivates License (http://creativecommons.org/licenses/by-nc-nd/3.o/), which permits unrestricted use, distribution, and reproduction in any medium, provided the original work is properly cited. 